\title{
RESÍDUOS DE AGROTÓXICOS PIRETRÓIDES EM HORTALIÇAS *
}

\author{
MARIA TERESA PLATA OVIEDO ** \\ MARIA CECÍLIA DE FIGUEIREDO TOLEDO *** \\ EDUARDO VICENTE ****
}

\begin{abstract}
Foram analisados resíduos de Cipermetrina, Deltametrina e Permetrina nas seguintes hortaliças: alface, acelga, chicória, repolho, tomate, chuchu, batata, cenoura, mandioca e mandioquinha. As amostras foram coletadas no restaurante da Universidade Estadual de Campinas, no período de outubro/1998 a abril/2001. Também foram analisadas hortaliças comercializadas nas Centrais de Abastecimento de Campinas, coletadas no período de outubro/2000 a agosto/2001. Os resíduos dos agrotóxicos piretróides foram determinados por cromatografia a gás com detector de captura de elétrons (CG-DCE-63Ni) e a confirmação da identidade dos picos realizada por cromatografia a gás acoplada em espectrômetro de massas (CGEM). Os resultados evidenciaram o uso inadequado desses piretróides, sendo detectados resíduos de Permetrina $(2,0 \mathrm{mg} / \mathrm{kg})$ em duas amostras de alface. Em tomate, resíduos de Permetrina (8,6 - 18,8 mg/ $\mathrm{kg})$ foram confirmados em três amostras e de Cipermetrina $(3,0 \mathrm{mg} / \mathrm{kg})$ em uma amostra. Em relação à Permetrina, os níveis encontrados em alface e tomate situaram-se acima dos limites máximos de resíduos (LMR) permitidos pela legislação vigente $(0,1 \mathrm{mg} / \mathrm{kg}$ em alface e $0,3 \mathrm{mg} / \mathrm{kg}$ em tomate). Quanto à Cipermetrina, sua presença foi detectada em tomate, embora seu uso seja autorizado somente para as culturas de amendoim, cebola e fumo. Resíduos de Deltametrina não foram encontrados nas amostras analisadas. Esses resultados indicam que as boas práticas agrícolas não estão sendo cumpridas por alguns produtores, sugerindo a necessidade de implementação de programa de monitoramento de piretróides em alface e tomate.
\end{abstract}

PALAVRAS-CHAVE: AGROTÓXICOS-RESÍDUOS; PIRETRÓIDES; HORTALIÇAS.

* Doutoranda em Ciência de Alimentos, Faculdade de Engenharia de Alimentos, Universidade Estadual de Campinas (UNICAMP), Campinas, SP (e-mail:matepo@hotmail.com).

** Professora, Doutora, FEA, UNICAMP, Campinas, SP (e-mail:macecil@fea.unicamp.br).

*** Pesquisador Científico, Instituto de Tecnologia de Alimentos (ITAL), Campinas, SP (e-mail: evicente@ital.org.br). 


\section{INTRODUÇÃO}

Os piretróides têm sido considerados como uma das classes de inseticidas com maior potencial na agricultura devido curta persistência no meio ambiente, alta atividade para insetos e baixa toxicidade para mamíferos. Entre os compostos sintéticos comercialmente disponíveis pertencentes a essa classe destacam-se pela sua utilização na agricultura Bifenato, Cipermetrina, Deltametrina, Fenpropatrina, Fenvalerato e Permetrina (MIDIO e MARTINS, 2000).

A aplicação de Cipermetrina é autorizada em partes aéreas de culturas de fumo, cebola e amendoim. A Deltametrina é permitida em bulbos, cereais, frutas, hortaliças folhosas e não-folhosas, leguminosas, raízes e tubérculos, sementes de oleaginosas, outros produtos como grãos armazenados e emprego domissanitário. Já a Permetrina é autorizada em culturas de cereais, hortaliças folhosas e não-folhosas, sementes de oleaginosas, grãos armazenados e emprego domissanitário (ANVISA, 2002).

Segundo informações obtidas na Seção de Resíduos de Pesticidas do Instituto Biológico de São Paulo (FERREIRA, 1998 e GEBARA, 2000), no Laboratório de Resíduos de Pesticidas do Instituto de Tecnologia de Alimentos (OLIVEIRA, 2003) e na Coordenadoria de Assistência Técnica Integral (GROPPO, 1998) de Campinas, existem poucos dados disponíveis sobre a presença de resíduos de piretróides em alimentos no Brasil, embora esses sejam bastante utilizados em hortaliças. A pouca disponibilidade de dados de resíduos de piretróides em alimentos deve-se a não inclusão de sua análise em programas de monitoramento conduzidos por diferentes instituições. Embora o Instituto Biológico tenha realizado pesquisas que demonstraram a presença de piretróides em alimentos, os dados gerados não podem ser publicados por estarem vinculados a projetos privados de pesquisa. A presença de resíduos de agrotóxicos acima dos limites permitidos ou em culturas não autorizadas pode originar não somente problemas de saúde pública e de ordem ecológica, como também barreira comercial, tanto no mercado interno como no externo.

A presente pesquisa teve como objetivo determinar os níveis residuais de Cipermetrina, Deltametrina e Permetrina em amostras de hortaliças cruas, servidas com maior frequência nos restaurantes universitários da Universidade de Campinas (UNICAMP) e de hortaliças comercializadas nas Centrais de Abastecimento de Campinas (CEASA).

\section{MATERIAL E MÉTODOS}

\subsection{AMOSTRAS, COLETA E ARMAZENAMENTO}

Foram coletadas no restaurante da UNICAMP amostras de $2 \mathrm{~kg}$ de alface, acelga, chicória, repolho, tomate e mandioquinha, além de amostras de $5 \mathrm{~kg}$ de chuchu, batata, cenoura e mandioca. A coleta foi efetuada no período de outubro/1998 a abril/2001, de acordo com amostragem mínima necessária para a análise de resíduos de agrotóxicos em vegetais in natura (YOKOMIZO et al., 1984). As amostras foram acondicionadas em sacos de polietileno e transportadas em caixa de isopor com gelo, devidamente rotuladas e enviadas ao laboratório de análises. Após homogeneização em multiprocessador de alimentos (Walita), as amostras foram acondicionadas em frascos de vidro de $500 \mathrm{~mL}$, fechados com tampas envolvidas com papel alumínio e armazenadas em congelador a $-18^{\circ} \mathrm{C} \pm 2^{\circ} \mathrm{C}$ até o momento da análise. Todas as análises foram realizadas em duplicata.

Para efeito de comparação foram também coletadas e analisadas 78 amostras das mesmas hortaliças, comercializadas nas Centrais de Abastecimento de Campinas no período de outubro/2000 a agosto/2001 (10 coletas mensais consecutivas). 


\subsection{PADRÕES E REAGENTES}

Foram preparados em acetona grau pesticida (Mallinckrodt) soluções de $200 \mathrm{mg} / \mathrm{L}$ dos padrões de Cipermetrina, Deltametrina e Permetrina (Agrevo Hoechst do Brasil) com 92,6, 98,8 e 93,5\% de pureza, respectivamente. A partir destas soluções foram obtidas, por diluição em hexano (p.r. Mallinckrodt), soluções contendo 5,15 mg/L e 25,75 mg/L de Cipermetrina, 5,22 mg/L e 26,1 mg/L de Deltametrina e $5,77 \mathrm{mg} / \mathrm{L}$ e $25,96 \mathrm{mg} / \mathrm{L}$ de Permetrina. Os demais reagentes utilizados foram $\mathrm{Na}_{2} \mathrm{SO}_{4}$, $\mathrm{NaCl}$ e florisil (p.r. Mallinckrodt), tratados previamente em mufla durante 6 horas a $600^{\circ} \mathrm{C}$, peneirados, guardados em frasco âmbar e conservados em dessecador com sílica gel até o momento da análise.

\subsection{MÉTODO DE ANÁLISE}

Os resíduos de inseticidas piretróides foram determinados pelo método de multirresíduo n.970.52 da AOAC (1995), modificado por PANG et al. (1995), para determinação de resíduos piretróides em frutas, vegetais e grãos. O procedimento empregado envolveu as etapas de extração e partição com acetonitrila, seguidas de limpeza em coluna de florisil.

\subsubsection{Método 970.52 da AOAC modificado por PANG et al. (1995)}

\section{a) Extração da amostra}

Aproximadamente 50 gramas de amostra, previamente homogeneizadas, foram extraídas com $150 \mathrm{~mL}$ de acetonitrila p.r. e agitadas por 30 minutos. O extrato foi filtrado em funil de Büchner com papel de filtro Whatman no 5 (tratado com acetona p.a. em Soxhlet durante 8 horas) e recolhido em kitasato acoplado à bomba de vácuo. Todo o material usado na filtragem foi enxaguado 2 vezes com $25 \mathrm{~mL}$ de acetonitrila p.r. O filtrado foi transferido para funil de separação de $1000 \mathrm{~mL}$ e o kitasato lavado 2 vezes com $10 \mathrm{~mL}$ de acetonitrila p.r., também transferidos para o funil de separação. Foram adicionados $60 \mathrm{~mL}$ de hexano p.r., agitou-se o funil vigorosamente por 2 minutos, sendo acrescentados $200 \mathrm{~mL}$ de solução aquosa de $\mathrm{NaCl} 3 \%$ (p/v) seguido de agitação vigorosa por 30 segundos. Depois da separação das fases foi descartada a fase aquosa, sendo a de hexano filtrada em funil contendo $15 \mathrm{~g}$ de $\mathrm{Na}_{2} \mathrm{SO}_{4}$ (previamente tratado durante 6 horas a $600^{\circ} \mathrm{C}$ ). Coletou-se o extrato em balão de evaporação de fundo redondo de $250 \mathrm{~mL}$, o funil de separação foi enxaguado 2 vezes com $20 \mathrm{~mL}$ de hexano e os eluídos percolados em funil contendo $\mathrm{Na}_{2} \mathrm{SO}_{4}$.

\section{b) Partição com acetonitrila}

Evaporou-se o extrato em rotavapor a $40^{\circ} \mathrm{C}$ até secura completa e o resíduo, redissolvido com $10 \mathrm{~mL}$ de hexano, foi transferido para funil de separação de $125 \mathrm{~mL}$. O balão de evaporação de fundo redondo foi enxaguado 2 vezes com $10 \mathrm{~mL}$ de hexano p.r. e a solução transferida para o mesmo funil de separação (125 mL). Adicionaram-se $30 \mathrm{~mL}$ de acetonitrila saturada com hexano p.r. $(300: 100 \mathrm{v} / \mathrm{v})$ ao funil de separação, sendo a solução agitada vigorosamente por 5 minutos. Depois da separação das fases, a fase de acetonitrila foi transferida para outro balão de evaporação de fundo redondo de 250 $\mathrm{mL}$. A fase de hexano foi extraída por 2 vezes com $30 \mathrm{~mL}$ de acetonitrila saturada com hexano p.r. e agitada vigorosamente a cada 5 minutos. As duas fases de acetonitrila foram combinadas no mesmo balão de evaporação de fundo redondo $(250 \mathrm{~mL})$. Os extratos foram combinados, evaporados em rotavapor a $50^{\circ} \mathrm{C}$ e os resíduos redissolvidos em $5 \mathrm{~mL}$ de hexano p.r.

\section{c) Coluna aberta de florisil}

Preparou-se coluna cromatográfica de $2 \mathrm{~cm}$ de diâmetro interno e $30 \mathrm{~cm}$ de comprimento, empacotada com $1 \mathrm{~cm}$ de $\mathrm{Na}_{2} \mathrm{SO}_{4}$ e 12 gramas de florisil p.r. 100-120 mesh (tratado previamente em mufla a $600^{\circ} \mathrm{C}$ durante 6 horas e desativado com $5 \%$ de água desmineralizada em sistema Milli $Q$ - Millipore $\mathrm{v} / \mathrm{m}$ ). Após adição de mais $1 \mathrm{~cm}$ de $\mathrm{Na}_{2} \mathrm{SO}_{4}$, previamente tratado, passaram-se pela coluna 
$50 \mathrm{~mL}$ de hexano. Sem deixar a coluna secar, transferiu-se com pipeta Pasteur o extrato diluído em $20 \mathrm{~mL}$ de hexano para a coluna. A eluição foi feita com $170 \mathrm{~mL}$ do eluente a $6 \%$ (éter etílico:éter de petróleo 6:94, v/v), sendo o eluato recolhido em balão de $250 \mathrm{~mL}$ (aproximadamente $3 \mathrm{~mL} /$ minuto). Após concentração em rotavapor, no máximo a $40^{\circ} \mathrm{C}$, o resíduo foi diluído em volume adequado de hexano. Foram injetados em cromatográfo a gás $3 \mu \mathrm{L}$ do extrato diluído.

\subsubsection{Análise Cromatográfica}

As análises foram realizadas em cromatógrafo a gás VARIAN, modelo 3400, equipado com detector de captura de elétrons (CG-DCE-63 Ni) e coluna megabore VA-608 (30 m de comprimento, $0,53 \mathrm{~mm}$ de diâmetro interno, $0,83 \mu \mathrm{m}$ de filme, fase estacionária de fenil metil silicone). Usou-se integrador-processador, modelo CG-300, da Instrumento Científico Ltda. Injetaram-se $3 \mu \mathrm{L}$ da amostra no modo on-column, com programação de temperatura em $245^{\circ} \mathrm{C}$ (15 minutos), primeiro gradiente de $5^{\circ} \mathrm{C} / \mathrm{min}$ até $260^{\circ} \mathrm{C}$ ( 15 minutos), gás de arraste nitrogênio $\left(20 \mathrm{~mL} /\right.$ minuto), injetor a $250^{\circ} \mathrm{C}$ e detector a $300^{\circ} \mathrm{C}$. Cada análise durou 33 minutos e os tempos de retenção dos padrões injetados foram: Permetrina 4,64 min, Cipermetrina 6,59 min e Deltametrina 13,35 min.

Os ingredientes ativos (Cipermetrina, Deltametrina e Permetrina) foram identificados pela comparação dos tempos de retenção dos picos obtidos nas amostras e nos respectivos padrões analíticos. Para quantificação usou-se o método de padronização externa. Foram preparadas diluições em hexano p.r. nas seguintes concentrações de cada um dos piretróides: 0,01; 0,02;0,04;0,08 e $0,2 \mathrm{mg} / \mathrm{L}$ e construídas curvas padrão analíticas relacionando a área do pico com a concentração das soluções de mistura dos padrões. Os coeficientes de correlação para Cipermetrina, Deltametrina e Permetrina foram 0,9990, 0,9997 e 0,9992, respectivamente.

\subsubsection{Confirmação da Identidade}

\section{Cromatografia a gás acoplada em espectrômetro de massas (CG-EM)}

A confirmação da identidade dos piretróides Permetrina e Cipermetrina foi realizada por CG-EM, utilizando-se cromatógrafo a gás modelo HP 6890, amostrador automático HP 7683, acoplado a detector de massas HP 5973, operando com fonte de elétrons de 70 eV. Utilizou-se coluna capilar HP-50+ (30 $\mathrm{m}$ de comprimento, $0,25 \mathrm{~mm}$ de diâmetro interno, $0,25 \mu \mathrm{m}$ de filme, com $50 \%$ de fenil e $50 \%$ de metilpolissiloxano), hélio como gás de arraste e vazão constante de $1,3 \mathrm{~mL} / \mathrm{min}$. As injeções foram feitas no injetor de temperatura programável (PTV), operando no modo splitless, mantido a $70^{\circ} \mathrm{C}$ por 0,05 minuto e programado até $300^{\circ} \mathrm{C}$ na razão de $700^{\circ} \mathrm{C} / \mathrm{min}$, permanecendo nessa temperatura por 0,7 min. Após 0,7 minutos da injeção foi aberta a válvula de purga do injetor por 1 minuto, com vazão de $500 \mathrm{~mL} / \mathrm{min}$. O volume de amostra injetado foi de $1 \mu \mathrm{L}$. A coluna foi mantida a $70^{\circ} \mathrm{C}$ por 1 minuto, primeiro gradiente de $50^{\circ} \mathrm{C} /$ minuto até $250^{\circ} \mathrm{C}$, segundo gradiente de $5^{\circ} \mathrm{C} /$ minuto até $300^{\circ} \mathrm{C}$, por 10 minutos, sendo a temperatura do detector mantida em $285^{\circ} \mathrm{C}$. O tempo da análise foi de 16 minutos.

Para a identificação dos piretróides nas amostras, essas foram injetadas inicialmente no modo Varredura (SCAN). A análise foi repetida no modo Single lon Monitoring (SIM), devido grande quantidade de interferentes, que permitiu maior seletividade e, por conseguinte, aumentou a sensibilidade da análise. Alternativamente, a confirmação dos piretróides nas amostras foi feita pela comparação dos espectros de massas no modo SCAN com os espectros dos padrões e com os espectros da biblioteca NIST 98. Tal procedimento só foi possível nos casos em que a concentração dos piretróides mostrou-se suficiente para a obtenção de cromatogramas e espectros com boa relação sinal/ruído. Os parâmetros de aquisição de dados no espectrômetro de massas, utilizados para a confirmação dos agrotóxicos, estão descritos na Tabela 1. 


\section{TABELA 1 - CRITÉRIOS ESTABELECIDOS PARA A IDENTIFICAÇÃO DOS AGROTÓXICOS}

PIRETRÓIDES

\begin{tabular}{|c|c|c|}
\hline A grot $x$ icos & $\begin{array}{c}\text { Te } m \text { po } d e \\
\text { reten } a \quad(m \text { in })\end{array}$ & "ons selecionados (S IM)* \\
\hline$C$ is $-P$ e $r m$ e trin $a$ & 9,65 & $163,165,183$ \\
\hline Trans-Permetrin a & 9,78 & $163,165,183$ \\
\hline C ip e $r m$ e trin $a-1$ & 10,66 & 165,181 \\
\hline C ip e $r m$ e trin $a-2$ & 10,77 & 165,181 \\
\hline C ip e $r m$ e trin $a-3$ & 10,87 & 165,181 \\
\hline C ip e $r m$ e trin $a-4$ & 10,90 & 165,181 \\
\hline
\end{tabular}

${ }^{*}$ Coluna HP-50+ (30 m x 0,25 $\mu \mathrm{m}$ de diâmetro interno e 0,25 $\mu \mathrm{m}$ de filme), gás de arraste hélio, com vazão constante de 1,3 mL/min. Espectrômetro de massas operando em modo SIM-70 eV. * De acordo com FILLION et al. (1995).

\subsection{VALIDAÇÃO DO MÉTODO 970.52 MODIFICADO POR PANG et al. (1995)}

\subsubsection{Amostras, Coleta e Armazenamento}

Foram coletadas no restaurante da UNICAMP três amostras de $2 \mathrm{~kg}$ de repolho, tomate e batata in natura, sem lavar. Estas hortaliças foram escolhidas por representarem maior freqüência de consumo nos restaurantes da Universidade. As amostras foram homogeneizadas em multiprocessador de alimentos (Walita), acondicionadas em frascos de vidro e armazenadas em congelador a -18ํㅡ até o momento da análise. As análises foram realizadas em duplicata.

\subsubsection{Recuperação do Método}

Para os testes de recuperação, as amostras de repolho, tomate e batata foram fortificadas com 1 e $2 \mathrm{~mL}$ da mistura de soluções padrões preparadas em hexano com 4 níveis de fortificação $(0,10 ; 0,20 ; 0,50$ e $1,0 \mathrm{mg} / \mathrm{kg})$ dos inseticidas estudados. Os agrotóxicos foram identificados no cromatograma pela comparação dos tempos de retenção dos picos obtidos nas amostras fortificadas e não-fortificadas com os tempos de retenção absolutos dos padrões analíticos submetidos às mesmas condições de análise. Calculou-se a porcentagem de recuperação pela diferença entre as áreas dos picos com os mesmos tempos de retenção encontrados para amostras fortificada e não-fortificadas, dividida pela área do pico do agrotóxico padrão considerado e multiplicado por cem. Todas as análises foram realizadas em duplicata.

\subsubsection{Limites de detecção e quantificação do método}

Os limites de detecção do método (LD) foram calculados conforme proposto por LONG e WINEFORDNER (1983) e LEITE (1998), a partir de 3 vezes o desvio do sinal da linha de base ou ruído do detector. Os limites de quantificação do método (LQ) foram calculados como 10 vezes o desvio do sinal produzido pela linha de base ou ruído, obtido a partir das respectivas curvas de calibração de cada um dos padrões (MILLER e MILLER, 1993).

\section{RESULTADOS E DISCUSSÃO}

\subsection{LIMITES DE DETECÇÃO E QUANTIFICAÇÃO DO MÉTODO}

A Tabela 2 mostra os limites de detecção e de quantificação do método para repolho, tomate e batata. Para efeito de comparação são também apresentados os limites máximos de resíduos desses agrotóxicos autorizados nas diferentes hortaliças. 
O LQ obtido para Deltametrina mostrou-se inferior ao limite máximo de resíduo (LMR) permitido para repolho, tomate e batata. No caso da Permetrina, o LQ foi inferior ao LMR para repolho e tomate. Esse resultado evidencia a aplicabilidade do método da AOAC (1995) nas culturas pesquisadas.

\section{TABELA 2 - LIMITES DE DETECÇÃO E QUANTIFICAÇÃO DOS AGROTÓXICOS PIRETRÓIDES EM REPOLHO, TOMATE E BATATA}

\begin{tabular}{|c|c|c|c|}
\hline Agrot $x$ icos & L D $\quad(m \mathrm{~g} / \mathrm{kg})$ & $\mathrm{L} Q(\mathrm{~m} \mathrm{~g} / \mathrm{kg})$ & $L M R \quad(m g / k g)$ \\
\hline \multicolumn{4}{|l|}{ R e polho } \\
\hline C ip erm etrin a & 0,005 & 0,020 & $\mathrm{n} . \mathrm{a}$. \\
\hline De Ita m e trin a & 0,003 & 0,007 & 0,01 \\
\hline Permetrin a & 0,009 & 0,030 & 0,10 \\
\hline \multicolumn{4}{|l|}{ Tom a te } \\
\hline C ip e rm etrin a & 0,005 & 0,020 & n. a. \\
\hline De lta m etrin a & 0,003 & 0,007 & 0,03 \\
\hline Perm etrina & 0,009 & 0,030 & 0,30 \\
\hline \multicolumn{4}{|l|}{ B a tata } \\
\hline C iperm etrin a & 0,005 & 0,020 & $\mathrm{n} . \mathrm{a}$. \\
\hline De Ita metrin a & 0,003 & 0,007 & 0,01 \\
\hline Permetrin a & 0,009 & 0,030 & n. a. \\
\hline
\end{tabular}

LD = Limites de detecção. $L Q=$ Limites de quantificação. LMR =Limite Máximo de Resíduo, Legislação Brasileira (BRASIL, 2001). n.a. = não-autorizado.

\subsection{RECUPERAÇÕES DOS AGROTÓXICOS PIRETRÓIDES}

No estudo de recuperação dos agrotóxicos piretróides, os solventes foram eluídos em coluna cromatográfica com florisil p.r. e recuperados os piretróides Permetrina, Cipermetrina e Deltametrina em um único eluído (éter etílico: éter de petróleo 6:94 v:v). A Tabela 3 apresenta os valores de recuperação dos piretróides em repolho, tomate e batata. A recuperação média variou de 90,0 a 97,0\% em repolho, de 84,0 a $90,0 \%$ em tomate e de $82,0 \%$ a $95,0 \%$ em batata, com coeficientes de variação (CV) na faixa de 6,5 a 13,2\%. Conforme definição do FDA (1994) as recuperações médias em repolho, tomate e batata podem ser consideradas completas $(>80 \%)$. O coeficiente de variação mostrou-se inferior a $16 \%$, atendendo portanto ao recomendado para análises de resíduos de pesticidas em alimentos em níveis de $\mathrm{mg} / \mathrm{kg}$ (HORWITZ, 1980).

Embora o método utilizado por PANG et al. (1995) para determinação de resíduos de piretróides em frutas, vegetais e grãos recomendasse o uso de $4 \%$ de $(\mathrm{p} / \mathrm{v})$ de $\mathrm{NaCl}$ na etapa de extração, a utilização dessa concentração resultou na obtenção de 3 fases ao invés de 2 e, conseqüentemente, em baixa recuperação dos agrotóxicos piretróides (22\% a 63\%). Em vista disto, a quantidade de $\mathrm{NaCl}$ foi reduzida para $3 \%$, o que aumentou a recuperação para valores acima de $80 \%$.

\section{TABELA 3 - RECUPERAÇÃO DOS AGROTÓXICOS PIRETRÓIDES EM REPOLHO, TOMATE E} BATATA

\begin{tabular}{|c|c|c|c|c|}
\hline Agrot xicos & A mostras & $\begin{array}{c}\mathrm{Recupera} \\
(\%)\end{array}$ & $\begin{array}{c}\text { Estim a tiva do } D P \\
( \pm)\end{array}$ & $\begin{array}{l}\text { Coeficie nte de } \\
\text { Varia } 0(\%)\end{array}$ \\
\hline C ipermetrin a & & 92,0 & 12,1 & 13,2 \\
\hline D e Ita m e trin a & R e polho & 90,0 & 9,0 & 10,0 \\
\hline P e rm etrin a & & 97,0 & 9,0 & 9,3 \\
\hline C ip ermetrin a & & 84,0 & 11,0 & 13,0 \\
\hline De Ita m e trin a & Tom a te & 90,0 & 8,0 & 8,9 \\
\hline P e rm etrin a & & 89,0 & 9,0 & 10,1 \\
\hline C ip erm e trin a & & 95,0 & 11,0 & 11,6 \\
\hline De Ita metrin a & B a ta ta & 82,0 & 5,3 & 6,5 \\
\hline P e rm etrin a & & 94,0 & 10,0 & 10,1 \\
\hline
\end{tabular}

* Médias das recuperações correspondentes aos níveis de fortificação: 0,10; 0,20; 0,50 e 1,0 mg/kg (2 repetições). DP = Desvio Padrão. 


\subsection{DETERMINAÇÃO DE AGROTÓXICOS PIRETRÓIDES EM HORTALIÇAS}

A Tabela 4 mostra os resíduos de Cipermetrina, Deltametrina e Permetrina encontrados em diferentes hortaliças servidas nos restaurantes da UNICAMP. Em todas as amostras ( $n=51)$, os níveis dos agrotóxicos foram inferiores aos respectivos LD. Esses resultados sugerem a utilização de manejo integrado de pragas, cultura orgânica ou cuidados especiais na aplicação dos agrotóxicos por parte dos agricultores que fornecem hortaliças para a UNICAMP.

\section{TABELA 4 - RESÍDUOS DE AGROTÓXICOS PIRETRÓIDES EM HORTALIÇAS SERVIDAS NOS RESTAURANTES DA UNICAMP NO PERÍODO DE OUTUBRO/1998 A ABRIL/2001}

\begin{tabular}{|c|c|c|c|c|}
\hline Hortali as & $\mathrm{n}$ & C ipermetrina & $\begin{array}{c}\text { A G RO T XICOS }(\mathrm{mg} / \mathrm{kg}) \\
\text { Deltametrin a }\end{array}$ & Permetrin a \\
\hline A If a c e & 6 & n.d. & n.d. & n.d. \\
\hline A c e Ig a & 5 & n.d & n.d. & n.d. \\
\hline Chic ria & 5 & n.d & n.d. & n.d \\
\hline R e polho & 6 & n.d & n.d. & n.d. \\
\hline Tom a te & 9 & n.d. & n.d. & n.d. \\
\hline $\mathrm{Chuchu}$ & 3 & $n \cdot d$ & n.d. & $n \cdot d$. \\
\hline B a ta ta & 7 & n.d & n.d. & n.d. \\
\hline C e noura & 5 & n.d. & n.d. & n.d. \\
\hline M a n d io c a & 2 & n.d & $n \cdot d$. & $n \cdot d$ \\
\hline $\mathrm{M}$ a nd io qu in $\mathrm{h}$ a & 3 & n.d & n.d. & n.d \\
\hline
\end{tabular}

$\mathrm{n}$ = número de amostras; n.d. = não-detectado; limite de detecção = 0,005 mg/kg para Cipermetrina, 0,003 mg/kg para Deltametrina e $0,009 \mathrm{mg} / \mathrm{kg}$ para Permetrina.

O número de amostras variou em função da oferta das horatliças nas datas das coletas

Os resultados obtidos mostraram-se semelhantes aos encontrados em monitoramento de resíduos de piretróides realizado por ZANDONÁ e ZAPPIA (1993). Tais autores analisaram 523 amostras de produtos hortifrutigranjeiros comercializados no Estado do Paraná e não detectaram resíduos do inseticida Deltametrina.

O resultado das análises de hortaliças produzidas por outros agricultores e comercializadas na CEASA de Campinas constam da Tabela 5. Conforme pode ser observado não foram detectados resíduos de Deltametrina em qualquer das amostras analisadas. Em alface foram encontrados resíduos de Permetrina e em tomate resíduos de Cipermetrina e Permetrina, todos em níveis superiores aos LMR estabelecidos para essas culturas.

TABELA 5 - RESÍDUOS DE AGROTÓXICOS PIRETRÓIDES EM HORTALIÇAS COLETADAS NAS CENTRAIS DE ABASTECIMENTO DE CAMPINAS (CEASA) NO PERÍODO DE OUTUBRO/ 2000 A AGOSTO/2001

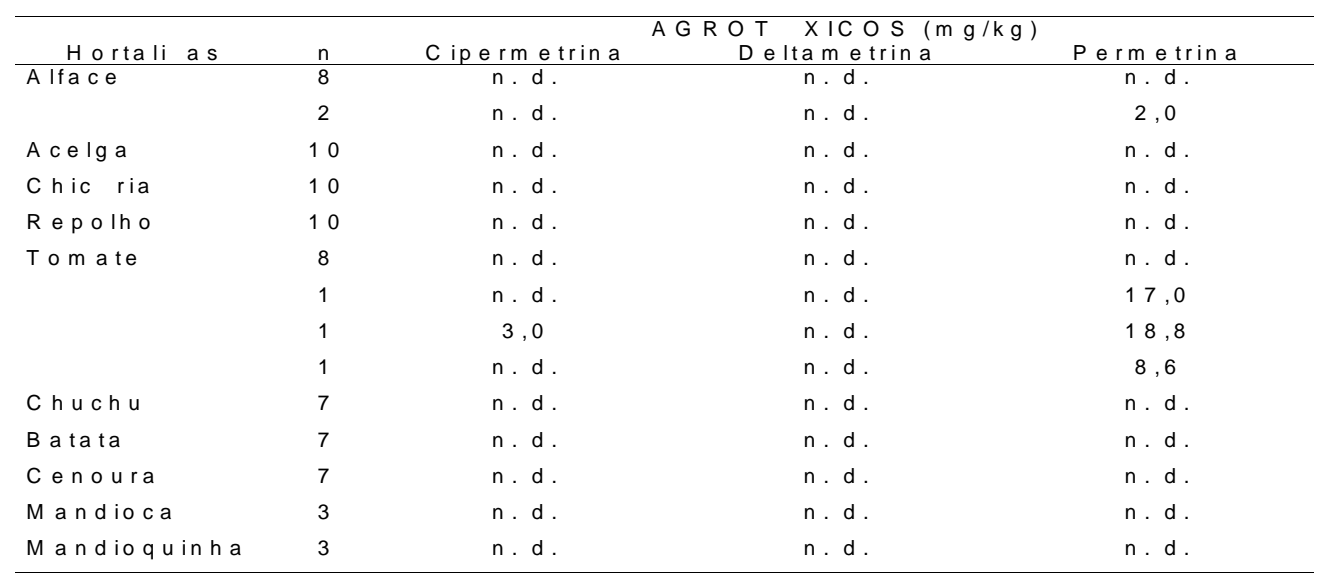

$\mathrm{n}=$ número de amostras; $\mathrm{n} . \mathrm{d} .=$ não-detectado; Limite de detecção = 0,005 mg/kg para Cipermetrina, 0,003 mg/kg para Deltametrina e $0,009 \mathrm{mg} / \mathrm{kg}$ para Permetrina. O número de amostras variou em função da oferta das hortaliças nas datas das coletas. 
A Figura 1 ilustra os cromatogramas correspondentes à mistura de padrões e às amostras de alface e tomate.

FIGURA 1 - CROMATOGRAMAS POR CG-DCE CORRESPONDENTES A: A) MISTURA DE PADRÕES 0,1 mg/mL DE PERMETRINA (P), CIPERMETRINA (C) E DELTAMETRINA (D), B) AMOSTRA DE ALFACE, C) E D) AMOSTRAS DE TOMATE
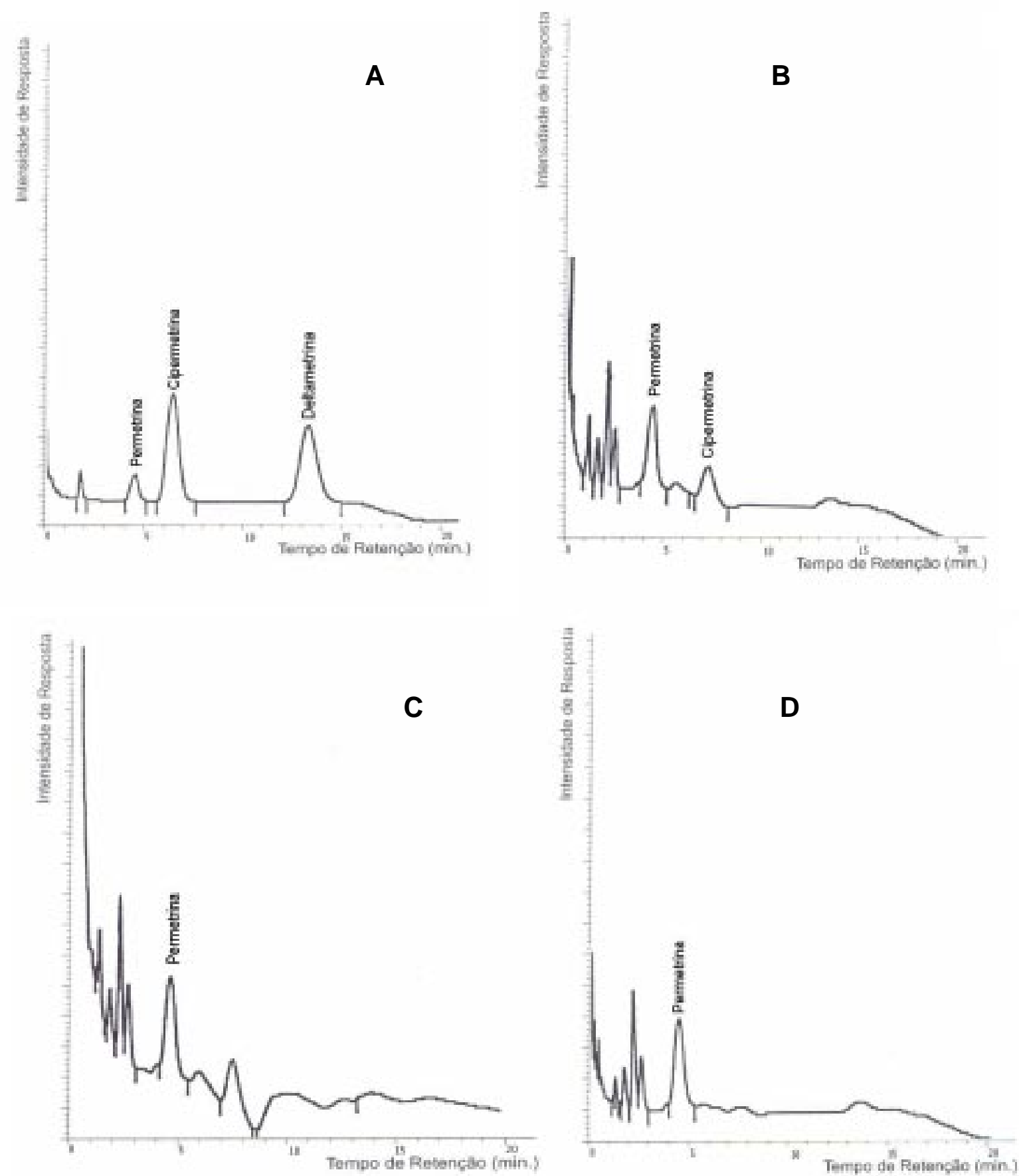

Condições de análise: coluna VA-608 (30 m x 0,53 mm di x 0,83 $\mu \mathrm{m}$ de filme, fase estacionária de fenil metil silicone); gás de arraste nitrogênio ( $20 \mathrm{~mL} / \mathrm{min})$; com programação de temperatura a $245^{\circ} \mathrm{C}(15 \mathrm{~min})$, primeiro gradiente de $5^{\circ} \mathrm{C} / \mathrm{min}$ até $260^{\circ} \mathrm{C}$ (15 min); injetor a $250^{\circ} \mathrm{C}$ e detector a $300^{\circ} \mathrm{C}$; volume de injeção $3 \mu \mathrm{L}$. 
No caso da Permetrina, cujo LMR em tomate é $0,3 \mathrm{mg} / \mathrm{kg}$, os níveis detectados e confirmados em três amostras $(8,6$ a $18,8 \mathrm{mg} / \mathrm{kg})$ foram aproximadamente 30 a 63 vezes superiores ao limite máximo autorizado. Níveis elevados de Permetrina, correspondentes a 20 vezes o LMR $(0,1 \mathrm{mg} / \mathrm{kg})$, também foram detectados em alface. A Cipermetrina, cujo uso não é autorizado em hortaliças, foi detectada em tomate $(3,0 \mathrm{mg} / \mathrm{kg})$. Conforme legislação vigente, o uso de Cipermetrina está autorizado somente para as culturas de fumo, cebola e amendoim. Quanto à Deltametrina, embora não tenha sido encontrada nas amostras analisadas, sua aplicação é autorizada para hortaliças folhosas e nãofolhosas. Os resultados obtidos evidenciaram duas situações irregulares, ou seja, a presença de resíduos de agrotóxicos acima dos limites máximos permitidos e seu uso em culturas não-autorizadas.

\section{CONCLUSÃO}

Os resultados obtidos indicaram que o método multirresíduo empregado é adequado para o monitoramento de Cipermetrina, Deltametrina e Permetrina nos alimentos estudados, já que os limites de quantificação mostraram-se inferiores aos limites máximos de resíduos estabelecidos pela legislação brasileira vigente.

As hortaliças oferecidas nos restaurantes da UNICAMP, no período de outubro/1998 a abril/2001, apresentaram resíduos de inseticidas piretróides em níveis inferiores aos limites de quantificação. As hortaliças disponíveis na CEASA de Campinas, no período de outubro/2000 a agosto/ 2001 evidenciram a presença de resíduos em níveis superiores ao limite máximo permitido e a aplicação de inseticida não-autorizado para a cultura. Esse panorama indica que as boas práticas agrícolas não estão sendo cumpridas por alguns produtores e evidencia a necessidade de implantação de programa de monitoramento de resíduos de piretróides em alimentos (particularmente em alface e tomate).

\section{ABSTRACT}

\section{PYRETHROID PESTICIDE RESIDUES IN VEGETABLES}

Residues of Cypermethrin, Deltamethrin and Permethrin were analysed in the following vegetables: lettuce, swiss chad, chicory, cabbage, tomato, chayote, potato, carrot, cassava and cassava specy. The samples were collected at the restaurant of the University of Campinas (UNICAMP), from October/1998 to April/2001. Vegetables comercialized at "CEASA" the "Centrais de Abastecimento de Campinas" from October/2000 to August/2001, were also analyzed. The pyrethroid residues were determined by gas chromatography with an electron capture detector (ECD-63Ni) and the pesticide identities confirmed by gas chromatography coupled to a mass spectrometer (GC-MS). The results showed that the pyrethroid pesticides were inappropriately used in some vegetables being detected Permethrin residues $(2.0 \mathrm{mg} / \mathrm{kg})$ in two samples of lettuce. In tomato, residues of Permethrin $(8.6 \mathrm{mg} / \mathrm{kg}-18.8 \mathrm{mg} / \mathrm{kg})$ were confirmed in three samples and of Cypermethrin $(3.0 \mathrm{mg} / \mathrm{kg})$ in one sample. The levels of Permethrin found in lettuce and tomato were above the maximum levels permitted (LMR) by the brazilian legislation (0.1 mg/kg in lettuce and $0.3 \mathrm{mg} / \mathrm{kg}$ in tomato). Cypermethrin residues were detected in tomato, although its use is only allowed in peanut, onion and tobacco. No Deltamethrin residues were detected in the samples analyzed. These results show that the good agricultural practices were not followed by some producers, suggesting the need to implement a monitoring program for the presence of pirethroides in tomato and lettuce.

KEY-WORDS: PESTICIDES-RESIDUES; PYRETHROID; VEGETABLES.

\section{REFERÊNCIAS}

1 ANVISA. Agência Nacional de Vigilância Sanitária. Disponível em: <http://www.anvisa.gov.br/legislacao.htm>. Acesso em 19 jan. 2002.

2 AOAC. Association of Official Analytical Chemists. Official methods of analysis of AOAC international. $15^{\text {th }}$ ed. Gaithersburg, 1995. (Method 970.52).

3 BRASIL. Ministério da Saúde. Relação de substâncias para uso fitossanitário e domissanitário: Portarias do ANVISA/Ministério da Saúde. 2.ed São Paulo: ILSI, 2000. 430 p.

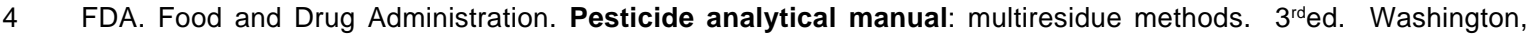
1994. v. 1, p. 103-302b. 

de 1998.

6 FILLION, J.; HINDLE, R.; LACROIX, M.; SELWYN, J. Multiresidue determination of pesticides in fruit and vegetables by gas chromatography-mass-selective detection and liquid chromatography with fluorescence detection. Journal of the Association of Official Analytical Chemists International, Arlington, v. 78, n. 5, p. 1252-1266, Set./Oct., 1995.

7 GEBARA, Amir B. Ocorrência de inseticidas piretróides em hortaliças. São Paulo: [s.n.], 12 de Abril de 2000.

8 GROPPO, Gerson A. Utilização de inseticidas piretróides em hortaliças. Campinas: [s.n.], 25 de Agosto de 1998.

9 HORWITZ, W.; KAMPS, L. R.; BOYER, K. W. Quality assurance in the analysis of food for trace constituents. Journal of the Association of Official Analytical Chemists, Arlington, v. 63, n. 6, p. 1344-1345, Nov., 1980.

10 LEITE, F. Sensibilidade, seletividade e limites de determinação e detecção. In: LEITE, F. Validação em análise química. 3.ed. Campinas: Átomo, 1998. Cap. 13, p. 67-69.

11 LONG, G. L.; WINEFORDNER, J. D. Limit of detection: a closer look at the IUPAC definition. Analytical Chemistry, Washington, v. 55, p. 712-724A, 1983.

12 MIDIO, A. F.; MARTINS, D. I. Agentes tóxicos contaminantes indiretos de alimentos. In: TOXICOLOGIA de alimentos. São Paulo: Varela, 2000. Cap. 4, p.163-252.

13 MILLER, J. C.; MILLER, J. N. Error in instrumental analysis; regression and correlation. In: MILLER, J. C.; MILLER, J. N. Statistic for analytical chemistry. $3^{\text {th }}$ ed. Chichester: Ellis Horwood, 1993. Chapter 5, p. 101-141.

14 OLIVEIRA, Jorge J. do Valle. Presença de resíduos de piretróides em alimentos. Campinas: ITAL, 2003.

15 PANG, G. F.; CHAO, Y. Z.; FAN, C. L.; ZHANG, J. J.; LI, X. M. Modification of AOAC multiresidue method for determination of synthetic pyrethroid residues in fruits, vegetables, and grains. Part I: Acetonitrile extraction system and optimization of florisil cleanup and gas chromatography. Journal of the Association of Official Analytical Chemists International, Arlington, v. 78, n. 6, p. 1481-1488, Nov./Dec., 1995.

16 YOKOMIZO, Y.; LARA, W. H.; PUGA, F.; BATISTA, G. C.; CARVALHO, P. R. N.; BARRETO, H. H. C.; MALTONE, C. A.; DOEGE, M. Resíduos de pesticidas em alimentos. Campinas: ITAL, 1984. 227 p.

17 ZANDONÁ, M. S.; ZAPPIA, V. R. S. Resíduos de agrotóxicos em alimentos: resultado de cinco anos de monitoramento realizado pela Secretaria de Saúde do Paraná. Pesticidas: Revista Técnico Científica, Curitiba, v. 3, n. 3, jan./dez., p. 49-95, 1993.

\section{AGRADECIMENTOS}

À FAPESP, pela concessão de bolsa de doutorado no país, Proc.97/09620-7, e reserva técnica correspondente, Proc.97/11887-1. Ao Instituto de Tecnologia de Alimentos (ITAL) de Campinas, pelo apoio e suporte técnico. 